\title{
4D Einstein-Gauss-Bonnet gravity with nonlinear electrodynamics: entropy, energy emission, quasinormal modes and deflection angle
}

\author{
S. I. Kruglov ${ }^{1}$ \\ Department of Physics, University of Toronto, \\ 60 St. Georges St., Toronto, ON M5S 1A7, Canada \\ Department of Chemical and Physical Sciences, University of Toronto, \\ 3359 Mississauga Road North, Mississauga, Ontario L5L 1C6, Canada
}

\begin{abstract}
The logarithmic correction to Bekenshtein-Hawking entropy in the framework of 4D Einstein-Gauss-Bonnet gravity coupled with nonlinear electrodynamics is obtained. We explore the black hole solution with the spherically symmetric metric. The logarithmic term in the entropy has a structure similar to the entropy correction in the semi-classical Einstein equations which mimics the quantum correction to the area low. The energy emission rate of black holes and energy conditions are studied. The quasinormal modes of a test scalar field are investigated. The gravitational lensing of light around BHs was studied. We calculated the deflection angle for some model parameters.
\end{abstract}

Keywords: Einstein-Gauss-Bonnet gravity; nonlinear electrodynamics; entropy; energy emission; quasinormal modes; deflection angle

\section{Introduction}

A modification of Einstein General Relativity (GR) by including higher order curvature terms in action is of interest because such terms can mimic quantum corrections. One of the ways is to add the Gauss-Bonnet (GB) term to Einstein-Hilbert action. Such theory, 4D Einstein-Gauss-Bonnet (EGB) gravity in four dimension, recently attracted much attention [1]-[17]). It was shown by Glavan and Lin [1] that 4D EGB theory gives non-trivial dynamics by the dimension regularization although the GB term is a topological invariant. In [18], [19] the authors obtained a solution of the semi-classical Einstein

\footnotetext{
${ }^{1}$ E-mail: serguei.krouglov@utoronto.ca
} 
equations with conformal anomaly that also is a solution in the regularized 4D EGB gravity. Recently, the regularization scheme of Glavan and Lin was debated in [20]-[26]. In particular, it was shown in [23], [24] that solutions in the 4D EGB theory differ from GR because they are due to extra infinitely strongly coupled scalar. It is worth noting that the scheme of [27], [28] gives the solution, in the spherically-symmetric metrics, that is a solution in the framework of dimensional regularization [1] (see [29]). Here, we explore a BH solution in the 4D EGB gravity theory coupled to nonlinear electrodynamics (NED) proposed in [30] making use of the regularization scheme of [1].

Because the first image of a super-massive black hole $(\mathrm{BH})$ in the center of M87 galaxy was received by the Event Horizon Telescope Collaboration [31] we investigate here the optical properties of $\mathrm{BH}$ by using the solution obtained in 4D EGB gravity coupled to NED. This paper is the continuation of the work [32]. NED considered here possesses the attractive features such as the absence of singularities and simplicity (the solution contains only elementary functions). In addition, at the weak field limit our NED is converted into Maxwell electrodynamics. It worth noting that the solution of well-known Born-Infeld electrodynamics in 4D EGB gravity contains special hypergeometric function [33]. The specific NED can give different astrophysical characteristics: the shadow radius of a charged $\mathrm{BH}$, the $\mathrm{BH}$ energy emission rate, and the deflection angle of light from the BH. Therefore it is of interest to test solutions of BHs in 4D EGB gravity coupled to different NED which effect on astrophysical characteristics. Thus, several BH solutions in 4D EGB gravity coupled to NED were studied [34]-[37]. In this paper we analyse the shadow, the energy emission rate, quasinormal modes and the light deflection angle of the magnetically charged BH by using NED proposed in $[30]$.

The paper is organized as follows. In Sec. 2, we consider the BH spherically symmetric solution in the framework of the 4D EGB gravity. It is shown that at infinity we have the Reissner-Nordström behavior of the charged BH and there is not a singularity at $r=0$. We obtained the logarithmic correction to Bekenstein-Hawking entropy in Sec. 3. In Sec. 4 we study the BH energy emission rate. The energy conditions are investigated in Sec. 5. It was shown that WEC, DEC and SEC are satisfied. In Sec. 6 we investigate the $\mathrm{BH}$ quasinormal modes and obtained the corresponding frequencies. We study the light defection angle by the BH solution in Sec. 7. Section 8 is a conclusion. 


\section{The model}

With the help of dimensional regularization of [1] the EGB gravity action in D-dimensions coupled to NED is

$$
I=\int d^{D} x \sqrt{-g}\left[\frac{1}{16 \pi G}\left(R+\frac{\alpha}{D-4} \mathcal{L}_{G B}\right)+\mathcal{L}_{N E D}\right]
$$

with $\alpha$ possessing the dimension of (length) ${ }^{2}$ and the NED Lagrangian, proposed in [30] is given by

$$
\mathcal{L}_{N E D}=-\frac{\mathcal{F}}{1+\sqrt[4]{2 \beta \mathcal{F}}}
$$

where the parameter $\beta(\beta \geq 0)$ has the dimension of (length $)^{4}, \mathcal{F}=(1 / 4) F_{\mu \nu} F^{\mu \nu}=$ $\left(B^{2}-E^{2}\right) / 2, F_{\mu \nu}$ is the strength tensor and the GB Lagrangian is

$$
\mathcal{L}_{G B}=R^{\mu \nu \alpha \beta} R_{\mu \nu \alpha \beta}-4 R^{\mu \nu} R_{\mu \nu}+R^{2} .
$$

The field equation corresponding to action (1) reads

$$
R_{\mu \nu}-\frac{1}{2} g_{\mu \nu} R+\frac{\alpha}{D-4} H_{\mu \nu}=-8 \pi G T_{\mu \nu}
$$

where $T_{\mu \nu}$ is the energy-momentum tensor and

$$
H_{\mu \nu}=2\left(R R_{\mu \nu}-2 R_{\mu \alpha} R_{\nu}^{\alpha}-2 R_{\mu \alpha \nu \beta} R^{\alpha \beta}-R_{\mu \alpha \beta \gamma} R_{\nu}^{\alpha \beta \gamma}\right)-\frac{1}{2} \mathcal{L}_{G B} g_{\mu \nu}
$$

The equations (1), (3)-(5) are defined in D dimensions. In the following, we consider the limit $D \rightarrow 4$. It was shown in [38] that to have a regular solution as $r \rightarrow 0$ one has to consider a magnetic BH. The magnetic energy density is given by

$$
\rho=-T_{t}{ }^{t}=-\mathcal{L}_{N E D}=\frac{q_{m}^{2}}{2 r^{3}\left(r+\sqrt{q_{m}} \beta^{1 / 4}\right)},
$$

where we have used the relation $\mathcal{F}=q_{m}^{2} /\left(2 r^{4}\right)$ and $q_{m}$ is a magnetic charge. The static and spherically symmetric space-time metric will be used. At the limit $D \rightarrow 4$ by exploring the regularization scheme of [1], the $t t$ component of Eq. (4) reads

$$
r\left(2 \alpha f(r)-r^{2}-2 \alpha\right) f^{\prime}(r)-\left(r^{2}+\alpha f(r)-2 \alpha\right) f(r)+r^{2}-\alpha=2 r^{4} G \rho .
$$


The equation (7) holds for any 4D EGB gravity model with the static and spherically symmetric metric. The general solution to Eq. (7) is given by

$$
f(r)=1+\frac{r^{2}}{2 \alpha}\left(1 \pm \sqrt{1+\frac{8 \alpha G}{r^{3}}\left(M+\int r^{2} \rho d r\right)}\right)
$$

where $M$ is the integration constant. For Maxwell electrodynamics the energy density is $\rho=q^{2} /\left(2 r^{4}\right.$ and Eq. (8) leads to the metric function obtained in [2]. But at the limit $r \rightarrow 0$ that solution leads to the non-physical complex value of the metric function. To have the stable $\mathrm{BH}$ [39] we will use the sign minus (the negative branch) before squire root in Eq. (8). For 4D EGB gravity coupled to NED (2) with the energy density (6), the solution (8) for the negative branch, gives the metric function [32]

$$
f(r)=1+\frac{r^{2}}{2 \alpha}\left(1-\sqrt{1+\frac{8 M \alpha G}{r^{3}}+\frac{4 \alpha q_{m}^{3 / 2} G}{\beta^{1 / 4} r^{3}} \ln \left(\frac{r}{r+\sqrt[4]{\beta q_{m}^{2}}}\right)}\right) .
$$

With the dimensionless variable $x=r / \sqrt[4]{\beta q_{m}^{2}}$, Eq. (9) is rewritten as

$$
f(x)=1+c x^{2}-c \sqrt{x^{4}+x\left(a+b \ln \left(\frac{x}{x+1}\right)\right)},
$$

where we use the dimensionless parameters

$$
a=\frac{8 M \alpha G}{\beta^{3 / 4} q_{m}^{3 / 2}}, \quad b=\frac{4 \alpha G}{\beta}, \quad c=\frac{\sqrt{\beta} q_{m}}{2 \alpha} .
$$

The asymptotic of the metric function $f(r)(9)$, for the negative branch, as $r \rightarrow \infty$ is given by

$$
f(r)=1-\frac{2 M G}{r}+\frac{G q_{m}^{2}}{r^{2}}+\mathcal{O}\left(r^{-3}\right) \quad r \rightarrow \infty .
$$

It follows from Eq. (12) that $M$ is a magnetic mass of the $\mathrm{BH}$. This equation shows the Reissner-Nordström behavior of the charged $\mathrm{BH}$ at large $r$, and the metric becomes flat at infinity $(r \rightarrow \infty)$. The asymptotic of the metric function $f(r)$, for the positive branch, does not correspond to the $\mathrm{BH}$ with the Reissner-Nordström behavior at infinity. We have the regular $\mathrm{BH}$ in our model because $\lim _{r \rightarrow 0} f(r)=1$. Therefore, there are not singularities in the model considered. 


\section{Thermodynamics and BH entropy}

By using the expression for Hawking temperature $T_{H}\left(r_{+}\right)=f^{\prime}\left(r_{+}\right) /(4 \pi)$, where $r_{+}$is the event horizon radius, $f\left(r_{+}\right)=0$, and the prime means the derivative with respect to the argument, we obtain [32]

$$
T_{H}\left(x_{+}\right)=\frac{\left(2 c x_{+}^{2}-1\right)\left(1+x_{+}\right)-b c^{2} x_{+}}{8 \pi \sqrt[4]{\beta q_{m}^{2}} x_{+}\left(1+x_{+}\right)\left(1+c x_{+}^{2}\right)},
$$

where $x_{+}=r_{+} / \sqrt[4]{\beta q_{m}^{2}}$. The BH gravitational mass, found from equation $f\left(x_{+}\right)=0$, reads

$$
M\left(x_{+}\right)=\frac{\beta^{3 / 4} q_{m}^{3 / 2}}{8 \alpha G}\left(\frac{1+2 c x_{+}^{2}}{c^{2} x_{+}}-b \ln \left(\frac{x_{+}}{x_{+}+1}\right)\right) .
$$

According to the first law of $\mathrm{BH}$ thermodynamics $d M\left(x_{+}\right)=T_{H}\left(x_{+}\right) d S+\phi d q$ ( $\phi$ is an electromagnetic potential) the entropy $S$ at the constant charge $q$ is given by

$$
S=\int \frac{d M\left(x_{+}\right)}{T_{H}\left(x_{+}\right)}=\int \frac{1}{T_{H}\left(x_{+}\right)} \frac{\partial M\left(x_{+}\right)}{\partial x_{+}} d x_{+} .
$$

Making use of Eqs. (13), (14) and (15) we obtain the entropy

$$
S=\frac{4 \pi \alpha}{G} \int \frac{1+c x_{+}^{2}}{x_{+}} d x_{+}=\frac{\pi r_{+}^{2}}{G}+\frac{4 \pi \alpha}{G} \ln \left(\frac{r_{+}}{\sqrt[4]{\beta q_{m}^{2}}}\right) .
$$

We put here the integration constant to be zero. Equation (16) shows that the entropy in our model besides the Bekenstein-Hawking area law includes the logarithmic correction. It should be noted that similar logarithmic term is also present in GR with a conformal anomaly, loop quantum gravity and string theory [18], [19], [40]. Thus, the logarithmic correction obtained mimics the quantum correction which is due to the GB Lagrangian. When the coupling constant of GB term $\alpha$ vanishes we come to the Bekenstein-Hawking entropy. For the massive BHs with the large event horizon radius $x_{+}$the leading term of the entropy is the Bekenstein-Hawking area law. But for small $x_{+}$(for the light BHs) the quantum corrections take place and the logarithmic term is important. It is worth noting that at some values of parameters $\alpha, \beta, q_{m}$ and $r_{+}$the entropy is zero. The event horizon radius, when the entropy vanishes, is the solution of equation $(S=0)$ 
$r_{+}^{4} \exp \left(r_{+}^{2} / \alpha\right)=\beta q_{m}^{2}$ which is $r_{0}=\sqrt{2 \alpha W_{0} \sqrt{\beta} q_{m} /(2 \alpha)}$, where $W_{0}(x)$ is the Lambert function. It is interesting that at $r_{+}<r_{0}$ the entropy becomes negative which can indicate a new type of instability. Probably, for such values of $r_{0}$ the $\mathrm{BH}$ does not exist. It is worth noting that the negative entropy of BHs was discussed in [41].

\section{The energy emission rate}

The particle emission rate of the $\mathrm{BH}$ depends on the model parameters $\alpha$, $\beta, q_{m}$ and $M$. The $\mathrm{BH}$ shadow can be connected with the high energy absorption cross section $\sigma$ for the observer located at infinity [42]-[44] (see also [45]). The absorption cross-section, at very high energies, oscillates around an approximate value of the photon sphere $\sigma \approx \pi r_{s}^{2}$, where $x_{s}$ defines the $\mathrm{BH}$ shadow radius $r_{s}=x_{s} \sqrt[4]{\beta q_{m}^{2}}$ (the impact parameter). The shadow radius can be expressed through the radius of the photon sphere $r_{p}$ by the relation $r_{s}=r_{p} / f\left(r_{p}\right)$ for a distant observer, and $r_{p}$ is the solution of the equation $f^{\prime}\left(r_{p}\right) r_{p}-2 f\left(r_{p}\right)=0$ [32]. The energy emission rate in the high energy is given by

$$
\frac{d^{2} E(\omega)}{d t d \omega}=\frac{2 \pi^{3} \omega^{3} r_{s}^{2}}{\exp \left(\omega / T_{H}\left(r_{+}\right)\right)-1},
$$

where $\omega$ denotes the emission frequency and $T_{H}$ is the Hawking temperature. With the help of the dimensionless variables $x=r / \sqrt[4]{\beta q_{m}^{2}}, \bar{T}_{H}\left(x_{+}\right)=$ $\sqrt[4]{\beta q_{m}^{2}} T_{H}\left(x_{+}\right)$, and Eq. (17), we obtain

$$
\beta^{1 / 4} \sqrt{q_{m}} \frac{d^{2} E(\omega)}{d t d \omega}=\frac{2 \pi^{3} \varpi^{3} x_{s}^{2}}{\exp \left(\varpi / \bar{T}_{H}\left(x_{+}\right)\right)-1},
$$

where the Hawking temperature is given by Eq. (13) and $\varpi=\beta^{1 / 4} \sqrt{q_{m}} \omega$. For some parameters $b$ at $a=5, c=1$ we obtain the event horizon radius, the photon sphere radius and the shadow radius (expressed via the dimensionless variables) presented in Table 1 (see also [32]). It is worth noting that the parameters $a, b$ and $c$ are connected with $M, \alpha, \beta$ and $q_{m}$ by Eq. (11). Because there are many parameters in the model, we use in Table 1 some set as an example. Making use of the data given in Table 1 we depicted the plot of the emission rate in Fig. 1 for $c=1, a=5$ and $b=1.5,2,2.5$. According 
Table 1: The event horizon, photon sphere and shadow dimensionless radii for $\mathrm{a}=5, \mathrm{c}=1$

\begin{tabular}{cccccccccccc}
\hline$b$ & 0.5 & 0.9 & 1.5 & 1.7 & 1.8 & 2 & 2.2 & 2.3 & 2.4 & 2.5 & 2.6 \\
\hline$x_{+}$ & 2.18 & 2.08 & 1.93 & 1.87 & 1.84 & 1.77 & 1.69 & 1.65 & 1.61 & 1.56 & 1.51 \\
\hline$x_{p}$ & 3.42 & 3.31 & 3.12 & 3.05 & 3.01 & 2.94 & 2.86 & 2.82 & 2.77 & 2.73 & 2.68 \\
\hline$x_{s}$ & 6.16 & 6.02 & 5.78 & 5.70 & 5.65 & 5.56 & 5.47 & 5.42 & 5.37 & 5.32 & 5.26 \\
\hline
\end{tabular}

to Fig. 1 there is a peak of the energy emission rate for the $\mathrm{BH}$ depending on model parameters. When the parameter $b$ increases, the maximum of the peak decreases and possesses the low frequency. Thus, the BH has a bigger lifetime at a bigger parameter $b$. One can investigate the dependence of the energy emission rate on parameters $\alpha, \beta, q_{m}$ and $M$, putting some numerical values for them.

\section{The energy conditions}

The symmetrical energy-momentum tensor with the spherically symmetry leads to $T_{t}{ }^{t}=T_{r}{ }^{r}$ and the radial pressure is given by $p_{r}=-T_{r}{ }^{r}=-\rho$. The tangential pressure is defined as $p_{\perp}=-T_{\vartheta}{ }^{\vartheta}=-T_{\phi}{ }^{\phi}$ so that [46]

$$
p_{\perp}=-\rho-\frac{r}{2} \rho^{\prime}(r),
$$

where the prime means the derivative with respect to the argument. The Weak Energy Condition (WEC) is satisfied when $\rho \geq 0$ and $\rho+p_{k} \geq 0$ $(\mathrm{k}=1,2,3)[47]$. This guarantees that the energy density is non-negative as measured by any local observer. In accordance with Eq. (6) $\rho \geq 0$. Making use of Eq. (6) one finds

$$
\rho^{\prime}(r)=-\frac{q_{m}^{2}\left(4 r+3 \beta^{1 / 4} \sqrt{q_{m}}\right)}{2 r^{4}\left(r+\beta^{1 / 4} \sqrt{q_{m}}\right)^{2}} \leq 0 .
$$

As a result, we have $\rho \geq 0, \rho+p_{r}=0, \rho+p_{\perp} \geq 0$ and WEC holds. The Dominant Energy Condition (DEC) takes place when [47] $\rho \geq 0, \rho+p_{k} \geq 0$, $\rho-p_{k} \geq 0$. These conditions include WEC and we have to verify the condition 


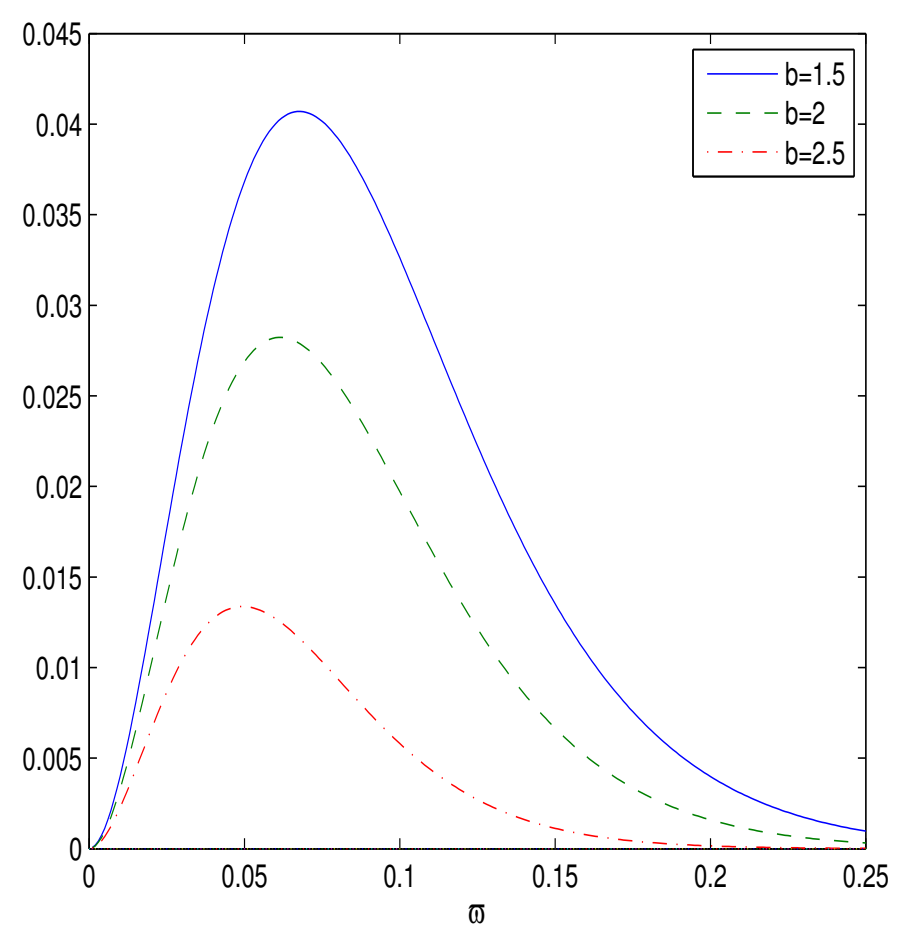

Figure 1: The plot of the function $\beta^{1 / 4} \sqrt{q_{m}} \frac{d^{2} E(\omega)}{d t d \omega}$ vs. $\varpi$ for $b=1.5,2,2.5$, $a=5, c=1$.

$\rho-p_{\perp} \geq 0$. From Eqs. (6), (19) and (20) we obtain

$$
\rho-p_{\perp}=\frac{q_{m}^{5 / 2} \beta^{1 / 4}}{4 r^{3}\left(r+\sqrt{q_{m}} \beta^{1 / 4}\right)^{2}} \geq 0 .
$$

Thus, DEC is satisfied. As a result, the sound speed cannot exceed the speed of light. The Strong Energy Condition (SEC) requires the condition $\rho+\sum_{k=1}^{3} p_{k} \geq 0$ [47]. With the help of Eqs. (6), (19) and (20) we obtain

$$
\rho+\sum_{k=1}^{3} p_{k}=2 p_{\perp}=\frac{q_{m}^{2}\left(2 r+\sqrt{q_{m}} \beta^{1 / 4}\right)}{2 r^{3}\left(r+\sqrt{q_{m}} \beta^{1 / 4}\right)^{2}} \geq 0,
$$

and SEC is satisfied. 


\section{Quasinormal Modes}

Quasinormal modes (QNMs) are characterised by complex frequencies $\omega$ which give an information about the stability of BHs under small perturbations and they do not depend on the initial conditions. The outgoing boundary condition is imposed at infinity and the ingoing boundary condition at the event horizon. If $\operatorname{Im} \omega>0$ the mode is unstable, otherwise it is stable. It was shown that Re $\omega$ in the eikonal limit is linked with the radius of the $\mathrm{BH}$ shadow [48], [49]. In addition, the real and imaginary parts of QNMs frequencies are connected with the angular velocity and Lyapunov exponent of unstable circular null geodesics [50]. The perturbations by a scalar massless field around BHs are characterized by the effective potential barrier

$$
V(r)=f(r)\left(\frac{f^{\prime}(r)}{r}+\frac{l(l+1)}{r^{2}}\right)
$$

where $l$ being the multipole number $0,1,2 \ldots$ Equation (23) can be represented in the terms of dimensionless variable $x=r / \sqrt[4]{\beta q_{m}^{2}}$ as

$$
V(x) \sqrt{\beta} q_{m}=f(x)\left(\frac{f^{\prime}(x)}{x}+\frac{l(l+1)}{x^{2}}\right) .
$$

The effective potential is plotted in Fig. 2 for $a=5, b=2, c=1$ and $l=1,2,3$ and for $a=5, c=1, l=1$ and $b=1,2,3$. According to Fig. 2 , Subplot 1, shows that the effective potentials represent a potential barrier with a maximum. The height of the potential increases when the $l$ increases. In accordance with Fig.2, Subplot 2, the height of the potential increases if the parameter $b$ increases. The real and imaginary parts of quasinormal frequencies are given by [48], [49]

$$
\operatorname{Re} \omega=\frac{l}{r_{s}}=\frac{l \sqrt{f\left(r_{p}\right)}}{r_{p}}, \quad \operatorname{Im} \omega=-\frac{2 n+1}{2 \sqrt{2} r_{s}} \sqrt{2 f\left(r_{p}\right)-r_{p}^{2} f^{\prime \prime}\left(r_{p}\right)},
$$

where $r_{s}$ is the $\mathrm{BH}$ shadow radius (the impact parameter), $r_{p}$ is the radius of the $\mathrm{BH}$ photon sphere, $n=0,1,2, \ldots$ is the overtone number. The real and imaginary parts of the frequencies versus the parameter $b$ at $a=5, c=1$, $n=1, l=5$ are given in Table 2 . The imaginary parts of the frequencies in Table 2 are negative, and therefore, the modes are stable and the real part represents the frequency of oscillations. According to Table 2 when 

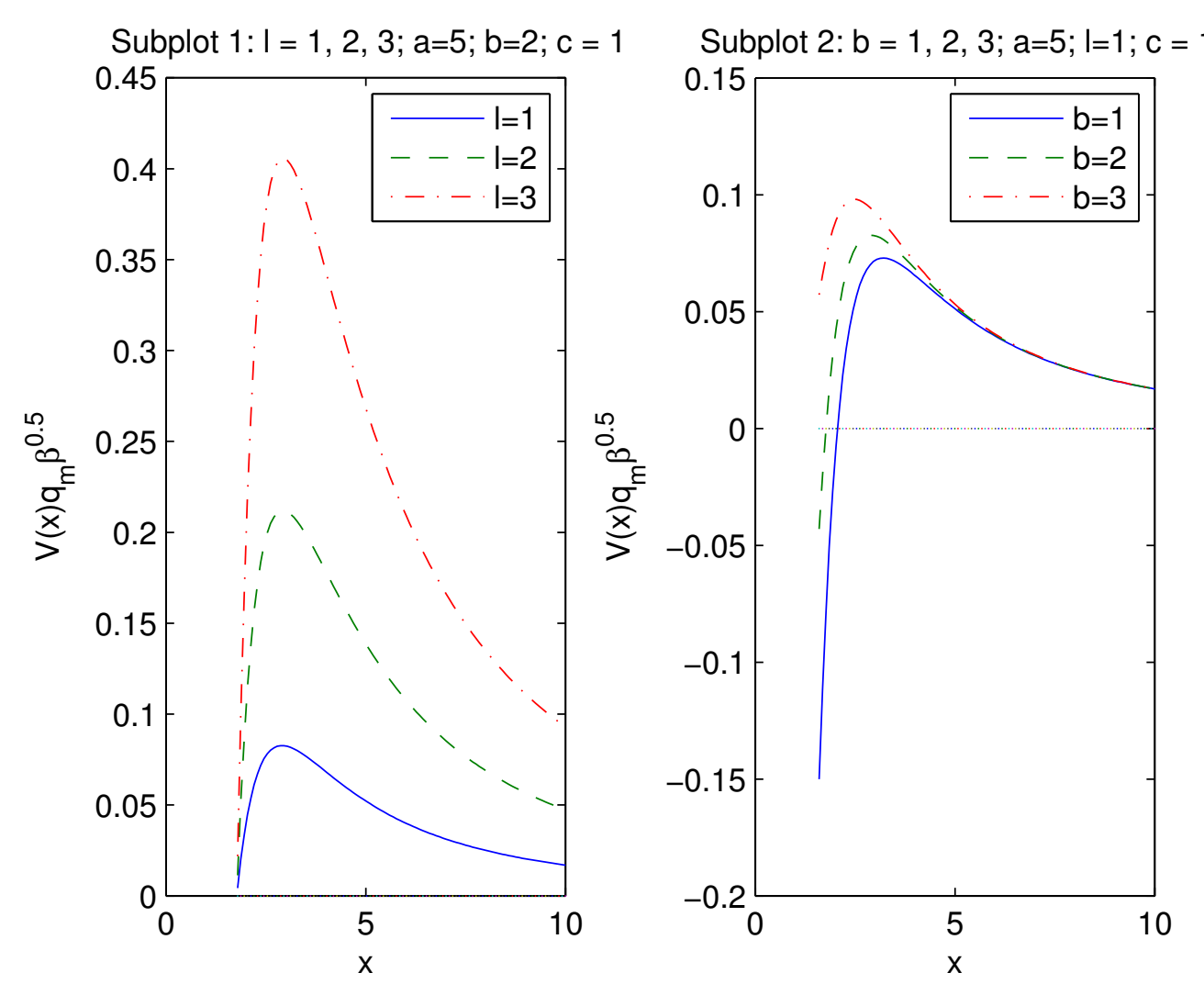

Figure 2: The plot of the function $V(x) \sqrt{\beta} q_{m}$ for $a=5, c=1$.

the parameter $b$ increases the real part of the reduced frequency $\sqrt[4]{\beta q_{m}^{2}} \operatorname{Re} \omega$ increases, but absolute value of the imaginary part of the reduced frequency $\left|\sqrt[4]{\beta q_{m}^{2}} \operatorname{Im} \omega\right|$ decreases. In other words, increasing the parameter $b$ the scalar perturbations oscillate with greater frequency and decay slowly. To study the dependence of frequencies on parameters $\alpha, \beta, M, q_{m}$ one has to put numerical numbers for these parameters in Eq. (25). 
Table 2: The real and the imaginary parts of the frequencies vs the parameter $b$ at $n=1, l=5, a=5, c=1$

\begin{tabular}{cccccccc}
\hline$b$ & 1.5 & 1.7 & 2 & 2.2 & 2.4 & 2.5 & 2.6 \\
\hline$\sqrt[4]{\beta q_{m}^{2}} \operatorname{Re} \omega$ & 0.865 & 0.877 & 0.899 & 0.914 & 0.931 & 0.940 & 0.951 \\
\hline$-\sqrt[4]{\beta q_{m}^{2}} \operatorname{Im} \omega$ & 0.2212 & 0.2208 & 0.2202 & 0.2191 & 0.2170 & 0.2163 & 0.2149 \\
\hline
\end{tabular}

\section{Deflection angle}

Let us study the light deflection angle by the BH solution (9). We can determine the total deflection angle $\Delta \varphi$ by the formula [51] (see also [52])

$$
\Delta \varphi=2 \int_{r_{p}}^{\infty} \frac{d r}{r \sqrt{\frac{r^{2} f\left(r_{p}\right)}{r_{p}^{2}}-f(r)}}-\pi
$$

where $r_{p}$ is the photon sphere radius. Taking into account that $r_{p} / \sqrt{f\left(r_{p}\right)}=$ $r_{s}$ is the shadow sphere radius $\left(r_{s}=\xi\right.$ is the impact parameter) one can represent Eq. (26) in terms of the dimensionless variable as

$$
\Delta \varphi=2 \int_{x_{p}}^{\infty} \frac{d x}{x \sqrt{\frac{x^{2}}{x_{s}^{2}}-f(x)}}-\pi
$$

Making use of data in Table 1 we obtain the deflection angles from Eq. (27) represented in Table 3 . According to Table 3 when the parameter $b$

Table 3: The deflection angles for $\mathrm{a}=5, \mathrm{c}=1$

\begin{tabular}{cccccccccccc}
\hline$b$ & 0.5 & 0.9 & 1.5 & 1.7 & 1.8 & 2 & 2.2 & 2.3 & 2.4 & 2.5 & 2.6 \\
\hline$\Delta \varphi$ & 4.12 & 3.58 & 3.1 & 3.02 & 2.96 & 2.86 & 2.81 & 2.77 & 2.76 & 2.73 & 2.69 \\
\hline
\end{tabular}

increasing, with fixed $a$ and $c$, the deflection angle is decreased. One can also study the dependence of the deflection angle on parameters $\beta, \alpha, q_{m}$ and $M$ by taking the numerical values for these parameters and putting them in Eq. $(9)$, finding the solution for $r_{p}: 2 f\left(r_{p}\right)-r_{p} f^{\prime}\left(r_{p}\right)=0\left(r_{s}=r_{p} / \sqrt{f\left(r_{p}\right)}\right)$, and calculating the integral (26). 


\section{Conclusion}

We use the exact spherically symmetric and magnetically charged $\mathrm{BH}$ solution in 4D EGB gravity coupled to NED obtained in [32] for further investigations. It is shown that we have the Reissner-Nordström behavior of the charged $\mathrm{BH}$ at infinity and there are not singularities at the origin of the $\mathrm{BH}$ (at $r=0$ ). Therefore, our solution for the metric function describes regular BHs. The logarithmic correction to the Bekenstein-Hawking entropy was obtained from first law of BH thermodynamics. Similar corrections to the area law are appeared in quantum gravity. Thus, the logarithmic correction obtained mimics a quantum correction to the Bekenstein-Hawking entropy and it is due to the GB term in the action. If the GB parameter $\alpha$ vanishes $(\alpha=0)$ one comes to the Hawking area law. We obtained the solution for the event horizon radius when the entropy becomes zero. For the light BHs the logarithmic correction is important while for massive BHs (for a big event horizon radius) such correction is small. Then the energy emission rate of $\mathrm{BHs}$ was studied. We shown that the $\mathrm{BH}$ energy emission rate decreases with increasing the model parameter $b$ and the $\mathrm{BH}$ has a bigger lifetime. To verify that the energy density is positive as measured by any local observer and the sound speed does not exceed the light speed, we investigated the energy conditions. It was demonstrated that WEC, DEC and SEC are satisfied. The quasinormal modes that describe small perturbations around BHs were investigated. We studied the dependence of the hight of the effective potential barrier on the multipole number $l$ and model parameter $b$. The height of the potential increases when the 1 or $b$ increases. Complex frequencies where the real part represents the frequency of oscillations and imaginary part characterises the oscillation decay were calculated. We demonstrated that increasing the parameter $b$ the scalar perturbations oscillate with greater frequency and decay slowly. Then the gravitational lensing of light around BHs was studied by calculating the deflection angle $\Delta \varphi$ for some parameters. The deflection angle depends on the photon sphere radius $r_{p}$, shadow radius $r_{s}$ and model parameters. It was shown that $\Delta \varphi$ is decreased if the parameter $b$ increasing at fixed $a$ and $c$.

Thus, we studied the influence of the BH solution in 4D EGB gravity on optical behaviour of the BHs. 


\section{References}

[1] D. Glavan and C. Lin, Phys. Rev. Lett. 124, 081301 (2020).

[2] P. G. S. Fernandes, Phys. Lett. B 805135468 (2020).

[3] R. A. Konoplya and A. Zhidenko, Phys. Rev. D 101, 084038 (2020).

[4] K. Jusufi, Ann. Phys. 421, 168285 (2020).

[5] S. G. Ghosh, D. V. Singh, R. Kumar, and S. D. Maharaj, Ann. Phys. 424, 168347 (2021).

[6] S. G. Ghosh and S. D. Maharaj, Phys. Dark Univ. 30, 100687 (2020).

[7] R. Kumar and S. G. Ghosh, JCAP 07, 053 (2020).

[8] X. H. Jin, Y. X. Gao, and D. J. Liu, Int. J. Mod. Phys. D 29, 2050065 (2020).

[9] K. Jusufi, A. Banerjee, and S. G. Ghosh, Eur. Phys. J. C 80, 698 (2020).

[10] M. Guo and P. Li, Eur. Phys. J. C 80, 588 (2020).

[11] C. Zhang, S. Zhang, P. Li, and M. Guo, JHEP 08, 105 (2020).

[12] C-Y. Zhang, P-C. Li, and M. Guo, Eur. Phys. J. C 80, 874 (2020).

[13] S. Odintsov, V. Oikonomou, and F. Fronimos, Nucl. Phys. B 958, 115135 (2020).

[14] W. Ai, Commun. Theor. Phys. 72, 095402 (2020).

[15] P. G. Fernandes, P. Carrilho, T. Clifton, and D. J. Mulryne, Phys. Rev. D 102, 024025 (2020).

[16] B. E. Panah, K. Jafarzade and S. H. Hendi, Nucl. Phys. B 961, 115269 (2020).

[17] R. A. Hennigar, D. Kubiznak, R. B. Mann, and C. Pollack, JHEP 2020, 27 (2020).

[18] R. G. Cai, L. M. Cao, and N. Ohta, JHEP 1004, 082 (2010). 
[19] R.-G. Cai, Phys. Lett. B 733, 183 (2014).

[20] M. Gurses, T. C. Sisman, and B. Tekin, Phys. Rev. Lett. 125, 149001 (2020).

[21] M. Gurses, T. C. Sisman, and B. Tekin, Eur. Phys. J. C 80, 647 (2020).

[22] S. Mahapatra, Eur. Phys. J. C 80, 992 (2020).

[23] T. Kobayashi, JCAP 07, 013 (2020).

[24] J. Bonifacio, K. Hinterbichler, L. A. Johnson, Phys. Rev. D 102, 024029 (2020).

[25] J. Arrechea, A. Delhom, and A. Jiménez-Cano, Chin. Phys. C 45, 013107 (2021).

[26] M, Hohmann, C. Pfeifer, N. Voicu, Eur. Phys. J. Plus 136, 180 (2021).

[27] K. Aoki, M. A. Gorji, and S. Mukohyama, Phys. Lett. B 810, 135843 (2020).

[28] K. Aoki, M. A. Gorji, and S. Mukohyama, JCAP 2009, 014 (2020).

[29] K. E Jafarzade, M. K. Zangeneh, F. S. N. Lobo, Shadow, deflection angle and quasinormal modes of Born-Infeld charged black holes, 2010.05755 [gr-qc].

[30] S. I. Kruglov, Ann. Phys. (Berlin) 529, 1700073 (2017).

[31] K. Akiyama et al., Astrophys. J.875, L1 (2019); ibid L4 (2019).

[32] S. I. Kruglov, Symmetry 13, 204 (2021).

[33] K. Yang, B. M. Gu, S. W. Wei and Y. X. Liu, Eur. Phys. J. C 80, 662 (2020).

[34] A. Kumar., R. Kumar, Bardeen black holes in the novel 4D Einstein-Gauss-Bonnet gravity, arXiv:2003.13104 [gr-qc].

[35] A. Kumar, S. G. Ghosh, Hayward black holes in the novel 4D Einstein-Gauss-Bonnet gravity, arXiv:2004.01131 [gr-qc]. 
[36] D. V. Singh, R. Kumar, S. G. Ghosh and S. D. Maharaj, Ann. Phys. 424, 168347 (2021).

[37] S. G. Ghosh, D. V. Singh and S. D. Maharaj, Phys. Rev. D 97, 104050 (2018).

[38] K. A. Bronnikov, Phys. Rev. D 63, 044005 (2001).

[39] D. G. Boulware and S. Deser, Phys. Rev. Lett. 55, 2656 (1985).

[40] G. Cognola, R. Myrzakulov, L. Sebastiani, and S. Zerbini, Phys. Rev. D 88, 024006 (2013),

[41] M. Cvetic, S. Nojiri, and S. D. Odintsov, Nucl. Phys. B 628, 295 (2002).

[42] B. Mashhoon, Phys. Rev. D 7, 2807 (1973).

[43] C.W. Misner, K. S. Thorne, and J. A. Wheeler, Gravitation, Freeman, San Francisco (1973).

[44] Y. Decanini, G. Esposito-Farese, and A. Folacci, Phys. Rev. D 83, $044032(2011)$

[45] S. W. Wei and Y. X. Liu, JCAP 11, 063 (2013).

[46] I. Dymnikova, Class. Quant. Grav. 21, 4417 (2004).

[47] S. W. Hawking and G. F. R. Ellis, The large scale structure of spacetime, Canbridge Univ. Press (1973).

[48] K. Jusufi, Phys. Rev. D 101, 084055 (2020).

[49] K. Jusufi, Phys. Rev. D 101, 124063 (2020).

[50] V. Cardoso, A. S. Miranda, E. Berti, H. Witek and V. T. Zanchin, Phys. Rev. D 79, 064016 (2009).

[51] S. Weinberg, Gravitation and Cosmology: Principles and Applications of the General Theory of Relativity, Wiley, New York (1972).

[52] P. Kocherlakota and L. Rezzolla, Phys. Rev. D 102, 064058 (2020). 